\title{
Attractive forces between circular polyions of the same charge
}

\author{
Silvia Martins and Jürgen F. Stilck \\ Instituto de Fisica, Universidade Federal Fluminense \\ Av. Litorânea s/n, 24210-340, Niterói, RJ, Brazil
}

(November 19, 2018)

\begin{abstract}
We study two models of ringlike polyions which are two-dimensional versions of simple models for colloidal particles (model A) and for rodlike segments of DNA (model B), both in solution with counterions. The counterions may condense on $Z$ sites of the polyions, and we suppose the number of condensed counterions on each polyion $n$ to be fixed. The exact free energy of a pair of polyions is calculated for not too large values of $Z$, and for both models we find that attractive forces appear between the rings even when the condensed counterions do not neutralize the total charge of the polyions. This force is due to correlations between the condensed counterions and in general becomes smaller as the temperature is increased. For model A divergent force may appear as the separation between the rings vanishes, and this makes an analytical study possible for this model and for vanishing separation, showing a universal behavior in this limit. Attractive forces are found for model $\mathrm{A}$ if the valence of the counterions is larger than one. For model B, no such divergences are present, and attractive forces are found for a finite range of values of the counterion valence, which depends of $Z, n$, and the temperature.
\end{abstract}

05.70.Ce, 61.20.Qg, 61.25.Hq

\section{INTRODUCTION}

The interaction between colloidal particles in suspensions are a statistical mechanical problem which has attracted much attention from both theoretical and experimental points of view for more than one century now, but there are still issues which are not totally understood [1]. Besides the difficulty of properly taking into account the long range Coulomb interactions, it is necessary to consider the fact that each colloidal particle in solution may contribute with a quite large number of counterions, and their contribution to the thermodynamic properties of the suspension is quite important [2]. One particularly important issue related to these systems is the possibility of phase separation, and although it has been argued that phase separation may occur even in situations where repulsive interactions between pairs of colloidal particles are present [3], recently it was found that for aqueous solutions with monovalent counterions no phase separation is expected [4] and this seems to be consistent with conclusions drawn from simulations [5]. For multivalent counterions, however, correlations between counterions which are condensed on the colloidal particles may produce short range attractive effective interactions between the particles, even if their bare charge is not totally neutralized [6]. This interesting phenomenon of effective attractive interactions between objects with charges of the same sign has attracted much attention recently, particularly for systems of rodlike polyions, such as segments of DNA [7]

The theoretical approaches used so far to address the problem of attraction between rodlike polyions of the same charge may be classified in two groups. The first formulates the system as a field-theoretic problem and then studies its properties in a high temperature approximation [8]. The second approach, which is also approximate, supposes the system of polyions and condensed counterions to form a Wigner crystal at zero temperature, and then proceeds considering the effect of temperature on the system 9 13. Some time ago, a very simple model has been proposed for an isolated pair of rodlike polyions in a solution with counterions which may be exactly solved, with the aid of a computer, for not too large rods [10]. In this model, the only effect of the condensation of a counterion on the site where an ion is located is to renormalize the local charge. The valence of each counterion is $\alpha$, so that each counterion has a charge $\alpha q$, and the polyion consist of a linear array of $Z$ charges $-q$ with a distance $b$ between first neighbors. The solution has an effective dielectric constant $D$. The mean value of the number $n$ of condensed counterions may be determined by the Manning criterion [14] as

$$
n=\left(1-\frac{1}{\alpha \xi}\right) \frac{Z}{\alpha}
$$

where

$$
\xi=\frac{q^{2}}{D b k_{B} T}
$$

is the Manning parameter. For sufficiently low temperature $(\xi>1 / \alpha)$, counterions will therefore condense on the polyions. It should be remarked that the expression above for the number of condensed counterions is an approximation for an isolated polyion and in the limit $Z \rightarrow \infty$. One may wonder if it is still valid for finite values of $Z$ and, supposing that the Manning value for 
$n$ is the mean value of a distribution of numbers of condensed counterions it is relevant to ask if this distribution is broad or narrow. It turns out that the expression (11) is actually a good approximation even for rather small values of $Z$ and that in usual physical conditions the distribution of values of $n$ is quite narrow [15]. Now if we consider two polyions separated by a distance $d$, we may use expression (11) in the limits $d \rightarrow \infty$ and $d \rightarrow 0$. The total number of counterions condensed on both rods in the limit $d \rightarrow \infty$ will be

$$
n_{\infty}=2 n
$$

and if we now consider the other limit $d \rightarrow 0$ (where both chains merge into one with $Z$ charges $-2 q$ ) the number of condensed charges will now be given by

$$
n_{0}=\left(1-\frac{1}{2 \alpha \xi}\right) \frac{2 Z}{\alpha}
$$

and therefore the change in the number of condensed counterions is given by

$$
\Delta n=n_{0}-n_{\infty}=n_{\infty} \frac{1}{\alpha \xi-1}
$$

and for not too high temperatures it may be a reasonable approximation to ignore this change, as is done in the simple model we described.

In this paper we study two models for the interaction between two ringlike charge structures which are inspired by studies of colloidal particles and of rodlike polyions, respectively. The models are similar to the one for rodlike polyions described above, and we study the thermodynamic properties of these models by solving them exactly for not too large particles. In the first model (model A), the bare colloidal particles are supposed to be formed by $Z$ point charges $-q$ placed regularly on circles, and we consider two of these ringlike particles placed in a solution with counterions. The number of counterions condensed on each particle is equal to $n$ and we proceed calculating the force between them as a function of the separation between the rings, for fixed values of the valence $\alpha$ of the counterions and the Manning parameter $\xi$. The second model is a two-dimensional version for a model which was recently proposed for rodlike DNA segments, in which the negative charge of the polyion is supposed to be concentrated at the axis of a cylinder, while the counterions are condensed at the surface of this cylinder [16]. Thus, in model B we each ringlike polyion is formed by a point charge $-Z q$ in the center of the circle, while $n$ counterions may condense on $Z$ equally spaced sites on the circle. The basic point we address in our calculation is the possibility of attractive forces between polyions of the same total charge, and for both models we find that such forces are indeed possible.

In the next section we present both models in detail and the results we obtained for them, which are either analytical or numerically exact. Final comments and discussions may be found in section III.

\section{DEFINITION OF THE MODELS AND THEIR SOLUTION}

We consider two circular polyions of radius $r$ with an even number $Z$ of point charges $-q$ which are placed as follows:

- Model A Uniformly distributed on the circle.

- Model B In the center of the circle.

These polyions are in a ionic solution of $\alpha$-valent counterions, also supposed to be point charges (charge $\alpha q$ ). We will admit that $n$ of the counterions are condensed on $Z$ sites on the circle. In model A, since the counterions condense on top of the fixed negative charges, the only effect of a condensed counterion is to renormalize the charge on the condensation site, which will be equal to $q(\alpha-1)$. In model $\mathrm{B}$, the charge at an condensation site on the circle is equal to zero if no counterion is condensed on it and $\alpha q$ otherwise. The distance between the centers of the polyions is $2 r+d$. The definitions above are illustrated in figure 1 .

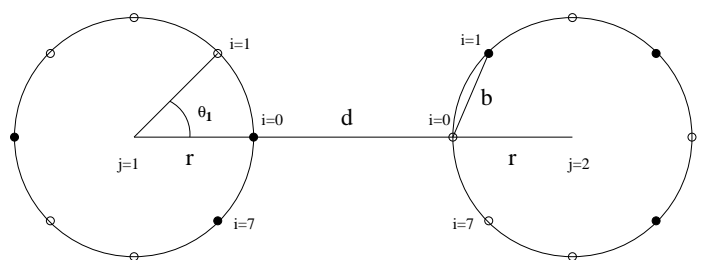

FIG. 1. A possible configuration of two ring polyions with $Z=8$ and $n=3$ condensed counterions. Full small circles indicate adsorption sites with condensed counterions, whereas empty small circles are empty condensation sites.

The positions of the condensation sites on the rings will be referred by two indices $j=1,2$, indicating the ring, and $i=0,1, \ldots, Z-1$, indicating the position on the ring. For model A, the electrostatic interactions between he charges may then be described by the Hamiltonian

$$
\mathcal{H}_{A}=\frac{q^{2}}{2 D r} \sum_{i, i^{\prime}=0}^{Z-1} \sum_{j, j^{\prime}=1}^{2} \frac{\left(1-\alpha \sigma_{i, j}\right)\left(1-\alpha \sigma_{i^{\prime}, j^{\prime}}\right)}{\delta\left(i, j, i^{\prime}, j^{\prime}\right)}
$$

where the sum is restricted to $(i, j) \neq\left(i^{\prime}, j^{\prime}\right)$ and the occupation variables $\sigma_{i, j}$ assume the value 0 if no counterion is condensed on site $(i, j)$ and 1 otherwise. $D$ is the dielectric constant and the ratio of the distance between two sites and $r$ is given by

$$
\begin{aligned}
& \delta\left(i, j ; i^{\prime}, j^{\prime}\right)= \\
& \begin{cases}2\left|\sin \frac{\theta_{i, i^{\prime}}}{2}\right| & \text { if } j=j^{\prime}, \\
\sqrt{\left(x+2-\cos \theta_{i}-\cos \theta_{i^{\prime}}\right)^{2}+\left(\sin \theta_{i}-\sin \theta_{i^{\prime}}\right)^{2}} & \text { otherwise; }\end{cases}
\end{aligned}
$$

where $x=d / r$ and 


$$
\begin{array}{r}
\theta_{i, i^{\prime}}=\frac{2 \pi\left(i-i^{\prime}\right)}{Z}, \\
\theta_{i}=\theta_{i, 0} .
\end{array}
$$

Model B may be described by the Hamiltonian

$$
\begin{gathered}
\mathcal{H}_{B}=\frac{q^{2} \alpha^{2}}{2 D r} \sum_{i, i^{\prime}=0}^{Z-1} \sum_{j, j^{\prime}=1}^{2} \frac{\sigma_{i, j} \sigma_{i^{\prime}, j^{\prime}}}{\delta\left(i, j, i^{\prime}, j^{\prime}\right)}- \\
\frac{Z q^{2} \alpha}{D r} \sum_{i=0}^{Z-1} \frac{\sigma_{i, 1}+\sigma_{i, 2}}{\delta(i)}+\frac{Z^{2} q^{2}}{r} \frac{1}{2+x},
\end{gathered}
$$

where the interaction between the central charge and the condensed counterions on the same ring was not included, since it is constant, and

$$
\delta(i)=\sqrt{1+(2+x)^{2}-2(2+x) \cos \theta_{i}}
$$

The partition function $Q$ is calculated summing over the possible positions of the condensed counterions

$$
Q_{A, B}=\sum_{\left\{\sigma_{i, j}\right\}}^{\prime} \exp \left(-\beta \mathcal{H}_{A, B}\right),
$$

where the prime denotes the constraint of having $n$ counterions condensed on each ring.

It is convenient to define adimensional hamiltonians $H$ through

$$
\beta \mathcal{H}_{A, B}=\xi^{\prime} H_{A, B},
$$

where $\xi^{\prime}=\beta q^{2} / D r$ is related to the Manning parameter defined in expression (1) for rodlike polyions. Then

$$
H_{A}=\frac{1}{2} \sum_{(i, j) \neq\left(i^{\prime}, j^{\prime}\right)} \frac{\left(1-\alpha \sigma_{i j}\right)\left(1-\alpha \sigma_{i^{\prime} j^{\prime}}\right)}{\delta\left(i, j, i^{\prime}, j^{\prime}\right)},
$$

and

$$
\begin{array}{r}
H_{B}=\frac{\alpha^{2}}{2} \sum_{(i, j) \neq\left(i^{\prime}, j^{\prime}\right)} \frac{\sigma_{i, j} \sigma_{i^{\prime}, j^{\prime}}}{\delta\left(i, j, i^{\prime}, j^{\prime}\right)} \\
-Z \alpha \sum_{i} \frac{\sigma_{i, 1}+\sigma_{i, 2}}{\delta(i)}+\frac{Z^{2}}{2+x} .
\end{array}
$$

We may define the statistical weights

$$
y_{i}=\exp \left(\frac{-\xi^{\prime}}{a_{i}}\right), i=1,2, \ldots, Z / 2,
$$

and

$$
z_{i}=\exp \left(\frac{-\xi^{\prime}}{b_{i}}\right), i=1,2, \ldots, m,
$$

related to the intra- and interpolyion interactions between charges at the condensation sites, respectively, and

$$
t_{i}=\exp \left(\frac{-\xi^{\prime}}{c_{i}}\right), i=0,1, \ldots, Z / 2,
$$

which correspond to the interaction between a central charge of a ring and the condensed counterions on the other ring. The denominators in the arguments of the exponentials are the adimensional distances between the interacting charges. For a pair of charges in the same polyion

$$
a_{i}=2\left|\sin \frac{\theta_{i}}{2}\right| .
$$

The expressions for the denominators $b_{i}$ are straightforward to obtain from (8) but will be omitted here for brevity. $m+1=Z^{2} / 4+Z / 2+1$ is the number of different distances between condensation sites located on distinct rings. Finally, the adimensional distances between condensation sites and the central charge of the other ring are $c_{i}=\delta_{i}$, according to equation (111).

Using these statistical weights, we may rewrite the partition function as

$$
Q_{A, B}=\sum_{i=1}^{N_{c}} \omega_{A, B}(i)
$$

where the sum runs over the

$$
N_{c}=\left[\frac{Z !}{n !(Z-n) !)}\right]^{2}
$$

allowed configurations of condensed counterions and

$$
\omega_{A}(i)=\prod_{j=1}^{Z / 2} y_{j}^{u_{A}(i, j)} \prod_{k=0}^{m} z_{k}^{v_{A}(i, k)} .
$$

The exponents $u_{A}(i j)$ and $v_{A}(i k)$ are quadratic polynomials in $\alpha$ with integer coefficients. For model B we obtain

$$
\omega_{B}(i)=\prod_{j=1}^{Z / 2} y_{j}^{u_{B}(i, j)} \prod_{k=0}^{m} z_{k}^{v_{B}(i, k)} \prod_{l=0}^{Z / 2} t_{l}^{w_{B}(i, l)},
$$

where $u_{B}(i j)$ and $v_{B}(i k)$ are integer coefficients multiplied by $\alpha^{2}$ and $w_{B}(i, l)$ are negative integer coefficients multiplied by $Z \alpha$. The whole set of integer coefficients can be calculated with the help of a computer program, and then we can obtain the partition function exactly for both models and not too large values of $Z$.

The force between the rings is given by

$$
F_{A, B}=-\frac{\partial \phi_{A, B}}{\partial d},
$$

where $\phi_{A, B}=-k_{B} T \ln Q_{A, B}$ is the Helmholtz freeenergy. It is convenient to define an adimensional force $f_{A, B}=\left(D r^{2} / q^{2}\right) F_{A, B}$, and thus

$$
f_{A, B}=\frac{1}{\xi^{\prime} Q_{A, B}} \frac{\partial Q_{A, B}}{\partial x} .
$$


For model A, we find that the force between the rings becomes attractive for short distances and $\alpha>1$. For the other cases the force is always repulsive. As $x \rightarrow 0$, the force is finite only for $\alpha=1$.

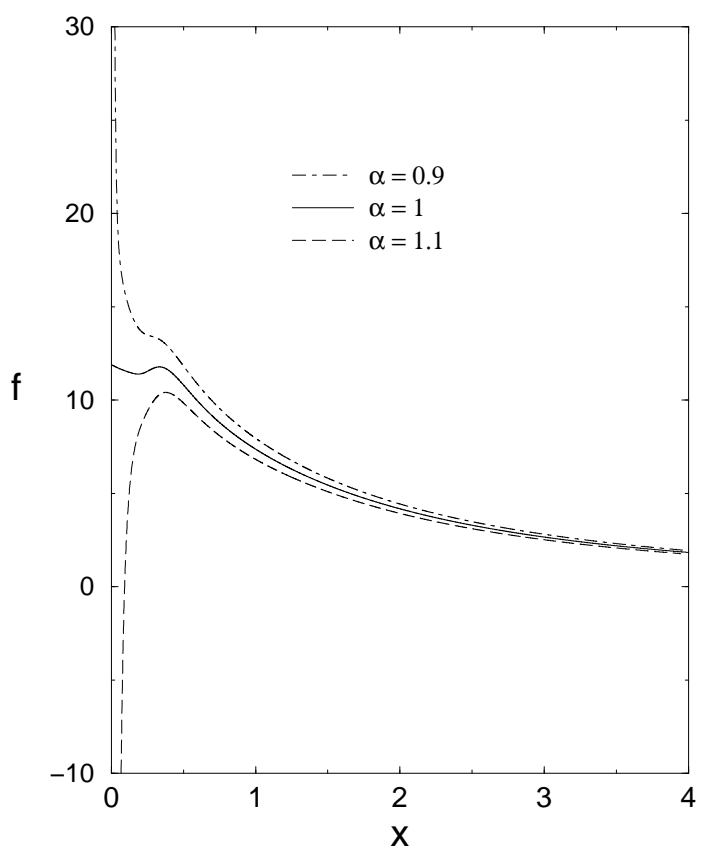

FIG. 2. The force between the rings as a function of the distance $x$ for model A. The results presented are for $Z=10$, $n=2$ and $\xi=2$.

The finite repulsive force observed in the limit $x \rightarrow 0$ when $\alpha=1$ may be easily understood. In this limit, the probability of having no charges condensed on the pair of sites $(0,1)$ and $(0,2)$ vanishes, since this would lead to a divergence in the energy. Thus this pair of sites does not contribute at all to the force, and the contributions of the other pairs is either positive or zero. This argument also explains the decreasing value of the force at vanishing distance as $n$ is increased, as may be seen in figure 3 .

To study behavior of the attractive force for model A at short distances $(\alpha>1)$, we consider the limit of small $x$. In this case, we have

$$
\lim _{x \rightarrow 0} z_{0}=0,
$$

where $z_{0}=\exp -\xi^{\prime} / x$, is related to the interaction between the charges in positions $(0,1)$ and $(0,2)$. All the other variables $y_{i}$ and $z_{i}$ are finite. We can therefore define the variable

$$
v=\min _{i}\left(v_{A}(i, 0)\right)
$$

where the index 0 refers to the pair of sites $(0,1)$ and $(0,2)$. We then rewrite the partition function as

$$
Q_{A}=z_{0}^{v} W(1-P),
$$

where

$$
W=\sum_{i=1}^{l} \prod_{j=1}^{Z / 2} y_{j}^{u_{A}(i, j)} \prod_{k=1}^{m} z_{k}^{v_{A}(i, k)},
$$

and

$$
P=\frac{1}{W} \sum_{i=l+1}^{N_{c}} z_{0}^{v_{A}(i, 0)-v} \prod_{j=1}^{Z / 2} y_{j}^{u_{A}(i, j)} \prod_{k=1}^{m} z_{k}^{v_{A}(i, k)} .
$$

In these equations, we considered the first $l$ configurations to have an exponent $v_{A}(i, 0)=v . P$ vanishes when $x \rightarrow 0$ since $v_{A}(i, 0)>v$ for $i \leq l+1$, so we may approximate

$$
Q_{A} \approx z_{0}^{v} W
$$

The possible values of $v_{A}(i, 0)$, are 1 if there are no condensed counterions in both sites, $1-\alpha$ when there is one condensed counterion on one of the sites, and $(1-\alpha)^{2}$ if there is a condensed counterion on each site. As we discussed before, the force becomes attractive only for $\alpha>1$, so we can conclude that the minimum value is $v=1-\alpha$. Using the above approximation for the partition function we obtain

$$
f=\frac{v}{x^{2}}+\frac{1}{\xi^{\prime} W} \frac{\partial W}{\partial x} .
$$

Taylor expanding the function $g(x)=\frac{1}{\xi^{\prime} W} \frac{\partial W}{\partial x}, g(x) \sim$ $g_{0}+g_{1} x+\ldots$ and keeping only the first term, we get

$$
f=\frac{v}{x^{2}}+g_{0}
$$

For the distance $x_{0}$ of vanishing force, we may then find the following scaling relation

$$
x_{0} \approx\left(\frac{\alpha-1}{g_{0}}\right)^{1 / 2}
$$

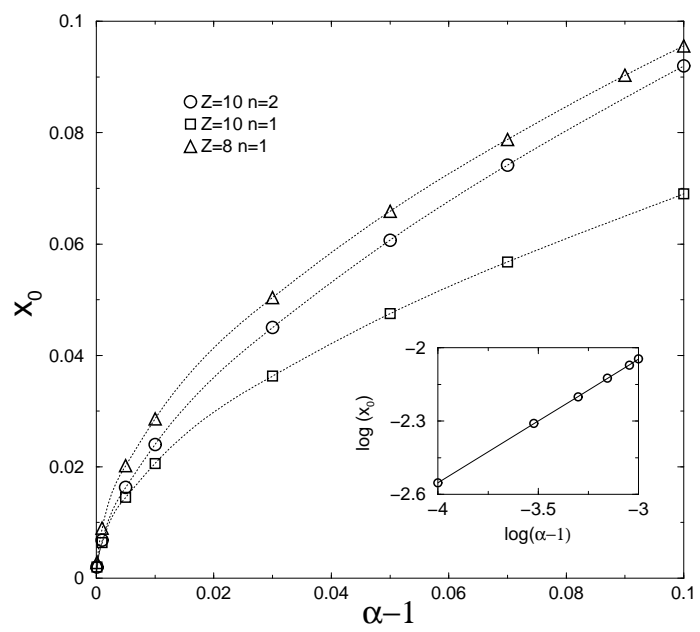

FIG. 3. The equilibrium distance $x_{0}$ as a function of $\alpha-1$ for $\xi^{\prime}=2$ (model A). The inset shows data for $Z=8, n=1$ in the region of $\alpha$ close to 1 . The scaling behavior is apparent and the numerical fit resulted in a value $0.51 \pm 0.01$ for the slope, in accordance with expression (34). 
In figure 3 curves of $x_{0}$ as a function of $\alpha$ are shown for some values of $Z, n$, and $\xi$, and the collapse of these curves at small values of $x_{0}$ may be seen. Also, in the inset the limiting behavior found in equation (34) is evident.

We proceed showing results for model B. In this case, it should be stressed that only in the rather unphysical limit of infinite temperature we have divergence in the force at vanishing values of $x$. These divergences are present for finite temperatures both in model $\mathrm{A}$ and in the similar model for rodlike polyions [10,17 and are due to pairs of sites with nonzero charge with vanishing distance between them. The absence of these divergences in model B prevents us from performing an asymptotic analysis similar to the one done for model $\mathrm{A}$, which would also prove the existence (or not) of attractive forces in model B. Simulations performed in a cylindrical version of model B found attractive forces at sufficiently low separation and enough condensed counterion charges 16].

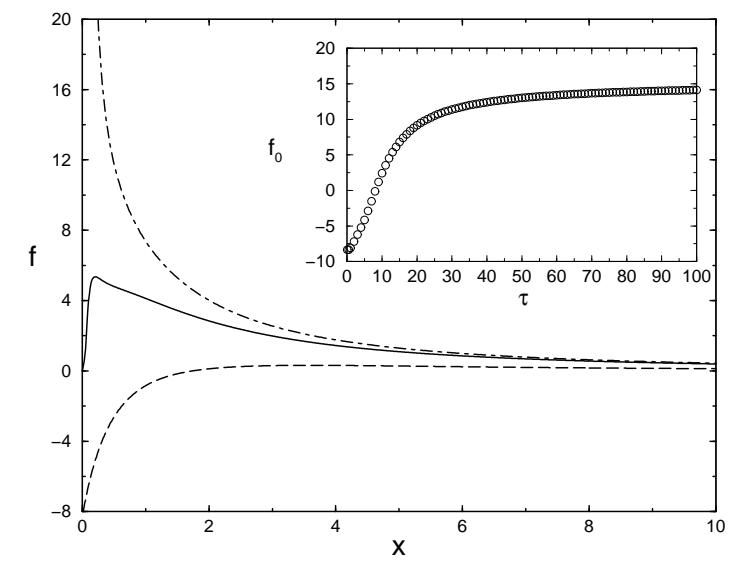

FIG. 4. The force as a function of the distance for model B. The results shown are for $Z=10, n=2$, and $\alpha=1.6$. Curves for three different values of the inverse temperature $\xi^{\prime}$ are shown: Dashed line $-\xi^{\prime}=2$; full line $-\xi^{\prime}=1.23$; dot-dashed line $-\xi^{\prime}=0$. In the inset, values of the force at vanishing $x\left(f_{0}=\lim _{x \rightarrow 0} f\right)$ are shown as a function of the reduced temperature $\tau=1 / \xi^{\prime}$.

In figure 1 the force between the rings for model B is shown as a function of the separation $x$. For sufficiently low temperatures and not too low or too high values of $\alpha$, attractive forces are found for low separation. As mentioned above, for finite temperatures the forces are always finite, since in this model the energy of the configurations where the pair of sites $(0,1)$ and $(0,2)$ is occupied diverges as $x \rightarrow 0$. The force at vanishing separation $f_{0}=\lim _{x \rightarrow 0} f(x)$ is shown as a function of the reduced temperature $\tau=1 / \xi^{\prime}$ in the inset of the figure, and increases monotonically, so that, as expected, it diverges to $+\infty$ as the temperature becomes infinite $\left(1 / \xi^{\prime} \rightarrow \infty\right)$. We obtained similar results for other values of $Z$ and $n$, so that the data in figure 1 are an example of a general trend.

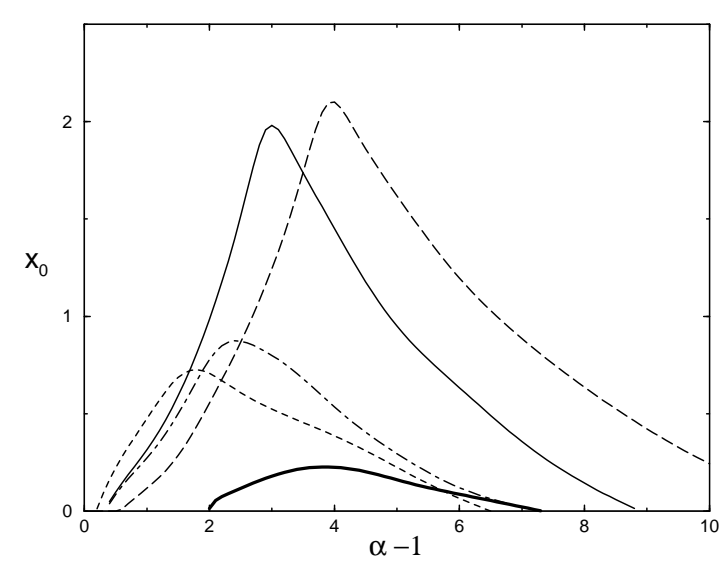

FIG. 5. The equilibrium distance $x_{0}$ as a function of $\alpha-1$ for different values of $Z, n$, and $\xi^{\prime}$ (model B). Full thin line - $Z=8, n=2$, and $\xi^{\prime}=1$; full thick line $-Z=8, n=2$, and $\xi^{\prime}=0.05$; long dashed line $-Z=10, n=2$, and $\xi^{\prime}=1$; short dashed line $-Z=8, n=3$, and $\xi^{\prime}=1$; dot-dashed line - $Z=10, n=3$, and $\xi^{\prime}=1$.

Figure 5 shows results for the equilibrium distance $x_{0}$ as a function of the valence $\alpha$ of the counterions for some choices of $Z, n$, and $\xi^{\prime}$. As mentioned above, we were not able to perform a simple analytical study of model $\mathrm{B}$ in the limit of vanishing $x$, and as expected no simple scaling behavior is found for model B in this limit, similar to the ones observed for model A and the other model proposed for rodlike polyions [10,17. Thus, the minimum value of $\alpha$ for the existence of an attractive interaction varies with $Z, n$, and $\xi^{\prime}$, and so does the initial slope of the curve $x_{0} \times \alpha$. Another feature of these curves is the existence of a maximum value of the valence of the counterions, above which no attractive force is found. Thus, as is apparent in figure 5, for each curve there are two values of $\alpha$ for which $x_{0}=0$. Results for $f_{0}$ as a function of $\alpha$ which will not be presented here for brevity also show this feature: The force is attractive in the interval between the limiting values of $\alpha$, and therefore passes through a minimum in some point inside the interval. It should be stressed, however, that the larger limiting value of $\alpha$ we found for model B assumes values which are too large to be physically realizable, but since the model itself is rather artificial, one may wonder of this effect may not be seen in more realistic models such as the one discussed in [16] and in experiments. For model $\mathrm{A}$ and the model which was proposed for rodlike polymers, no such maximum value of $\alpha$ is found, and the curves of $x_{0} \times \alpha$ pass through a maximum but approach zero asymptotically as $\alpha \rightarrow \infty$.

\section{FINAL COMMENTS AND DISCUSSIONS}

In an appropriate range of the parameters, both models studied here display attractive interaction forces, which may be explained by correlations between the counterions condensed on the ringlike polyions. As the 
temperature is increased, in general the correlations become weaker and therefore the attractive force decreases. In simplified models which were exactly solved before [10,17, as well as in model $\mathrm{A}$ in this work, the attractive forces increase without bond as the distance between the polyions is lowered, since one or more pairs of sites with opposite charges are separated by a distance which vanishes. This effect allowed an exact analysis of the models at vanishing separation, but also has the drawback of being not very reasonable physically. In particular, in the case of model A, it leads to the conclusion that it is sufficient to have only one condensed counterion on each polyion, with a valence slightly larger than one, to ensure the appearance of attractive forces. As was already found in simulations of cylindrical polyions [16 and other more realistic models for DNA segments 12], attraction between like charged polyions is not restricted to this rather artificial situation where sites with opposite charges become very close. In model B we find another example of this kind: The forces are finite for finite temperature, and a valence which is larger than one by a finite amount is needed to ensure attractive forces.

In both models studied here, when the parameters are such that attractive forces are found, the equilibrium distance $x_{0}$ as a function of $\alpha$ has a maximum. At low temperature, the maximum is sharp and located close to $\alpha=Z / n$, which corresponds to neutral polyions. As the temperature is increased, the maximum becomes broader and moves towards larger values of $\alpha$. With increasing temperature, the region of attractive force in the $\alpha \times x$ plane shrinks and finally disapears as the temperature becomes infinite. An example of this behavior is seen in the two curves for different temperatures for model B with $Z=8$ and $n=2$ in figure 5. Also, in figure 1 one notices that for infinite temperature $\left(\xi^{\prime}=0\right)$, the force is always repulsive.

\section{ACKNOWLEDGMENTS}

It is a pleasure to acknowledge Yan Levin for many suggestions in this work and for a critical reading of the manuscript. Partial financial support by the brazilian agencies CNPq and FAPERJ is gratefully acknowledged.

[1] D. G. Grier and J. C. Crocker, Phys Rev. E 61, 980 (2000).

[2] Y. Levin, M. C. Barbosa, and M. N. Tamashiro, Europhys. Lett. 41, 123 (1998); M. N. Tamashiro, Y. Levin, and M. C. Barbosa, Physica A 258, 341 (1998).

[3] R. van Roij and J. P. Hansen, Phys. Rev. Lett. 79, 3082 (1997); R. van Roij, M. Dijkstra, and P. Hansen, Phys. Rev. E 59, 2010 (1999).

[4] A. Diehl, M. C. Barbosa, and Y. Levin, Europhys. Lett. 53, 86 (2001).

[5] P. Linse and V. Lobaskin, Phys. Rev. Lett. 83, 4208 (1999); J. Chem. Phys. 112, 3917 (2000).

[6] M. J. Stevens and M. O. Robbins, Europhys. Lett. 12, 81 (1990); I. Rouzina and V. A. Bloomfield J. Phys. Chem. 100, 9977 (1996); Y. Levin, Physica A 265, 432 (1999); A. Diehl, M. N. Tamashiro, M. C. Barbosa, and Y. Levin, Physica A 274, 433 (1999).

[7] V.A. Bloomfield, Biopolymers 31, 1471 (1991); V.A. Bloomfield, Biopolymers 44, 269 (1997).

[8] B.-Y. Ha and A.J. Liu, Phys. Rev. Lett. 79, 1289 (1997).

[9] I. Rouzina and V. Bloomfield J. Phys. Chem. 100, 9977 (1996); B.I. Shklovskii, Phys. Rev. Lett. 82, 3268 (1999).

[10] J. J. Arenzon, J. F. Stilck, and Y. Levin, Eur. Phys. J. B 12, 79 (1999).

[11] F. J. Solis, and M. Olvera de la Cruz, Phys. Rev. E 60, 4496 (1999).

[12] A. A. Kornyshev and S. Leikin, Phys. Rev. Lett. 82, 4138 (1999).

[13] Y. Levin, J. J. Arenzon, and J.F. Stilck, Phys. Rev. Lett. 83, 2680 (1999).

[14] G. S. Manning, J. Chem. Phys. 51, 924 (1969); Q. Rev. Biophys. 11, 179 (1978).

[15] Y. Levin and M. C. Barbosa, J. Phys. II (France) 7, 37 (1997).

[16] A. Diehl, H. A. Carmona, and Y. Levin, Phys. Rev E 64, 1804 (2001).

[17] J. F. Stilck, Y. Levin and J. J. Arenzon, J. Stat. Phys (in press) (2002). 